\title{
INFALL OF A PARTICLE INTO A BLACK HOLE AS A MODEL FOR GRAVITATIONAL RADIATION FROM THE GALACTIC CENTER
}

\author{
Carlos O. Lousto \\ Department of Physics, University of Utah, Salt Lake City, UT 84112, USA
}

\begin{abstract}
I present here the results of the study of the gravitational radiation generated by the infall (from rest at radius $r_{0}$ ) of a point particle of mass $m_{0}$ into a Schwarzschild black hole of mass $M$. We use Laplace's transform methods and find that the spectra of radiation for $\sim 5 M<r_{0}<\infty$ presents a series of evenly spaced bumps. The total radiated energy is not monotonically decreasing with $r_{0}$, but presents a joroba (hunch-back) at around $r_{0} \approx 4.5 \mathrm{M}$. I finally discuss the detectability of the gravitational radiation coming from the black hole in the center of our galaxy.
\end{abstract}

\section{Perturbative approach}

Here I will report on work made in collaboration with R. Price (see Ref. 1 for further details.) The problem of a particle falling into a non-rotating black hole can be treated in the regime where the particle contributes perturbatively to the Schwarzschild metric. Thus, it was not surprising that soon after Zerilli (1970) wrote down his equation describing the propagation of gravitational waves on the Schwarzschild background, the case of a particle falling from infinity, both at rest and with a finite velocity, was solved using Fourier transform techniques. The key to the resolution to the problem of the infall from a finite distance (that had to wait 25 years) is the use of the Laplace's transform method instead. In this case, Zerilli's equation reads

$$
\frac{\partial^{2} \Psi_{\ell}}{\partial r *^{2}}+\left[\omega^{2}-V_{\ell}(r)\right] \Psi_{\ell}=-\dot{\psi}_{0}(r)+i \omega \psi_{0}(r)+S(r, \omega)
$$

where the $\ell$-multipole of the waveform is related to the metric components (in the Regge-Wheeler gauge) by

$$
\psi_{\ell}(r, t)=\frac{r}{\lambda+1}\left[K+\frac{r-2 M}{\lambda r+3 M}\left\{H_{2}-r \partial K / \partial r\right\}\right],
$$

where $\lambda \doteq(\ell+2)(\ell-1) / 2$. An important ingredient in Eq. (11) is the source term $S(r, \omega)$ that we had to compute for the particle infalling along a geodesic. We also had to consider the initial waveform $\psi_{0}(r)$ corresponding to a solution of the hamiltonian constraint. Here we have taken the particle limit of the Brill-Lindquist initial solution (the results do not differ notably had we chosen the Misner initial solution), and a time-symmetric situation $\left(\dot{\psi}_{0}(r)=0\right)$. 
Figure 1

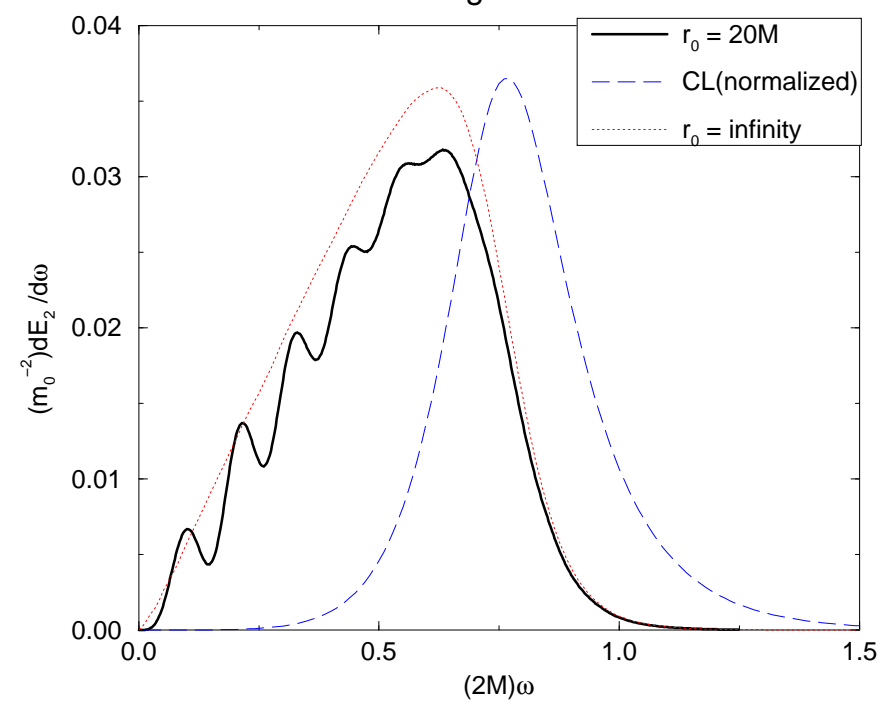

\section{Results}

To solve Eq. (1) we use the Green's function method, integrating over the total effective source, i.e. the right hand side of Eq. (11), to obtain the complex amplitude $A(\omega)$ of the outgoing radiation. The main results we have obtained can be summarized in the three figures we give here: In Fig. 1 the new feature is the appearance of bumps in the spectrum whose spacing decreases as $r_{0}$ increases. This can be understood as a consequence of the interference between the initial burst of radiation, soon after $t=0$, and the final one due to the infall of the particle into the black hole, In Fig. 2 the novelty is the joroba, with a local maximum at $r_{0}=4.5 \mathrm{M}$. This purely general relativistic effect can be attributed to the higher efficiency in the generation of radiation by the initial burst close to the maximum of the potential (located at $r \approx 3.1 M$.) Here is a small challenge for supercomputers: To check up to which mass ratio the joroba survives in the nonlinear regime. Fig. 3 shows the characteristic waveforms for different trajectories of the infalling particle. The main observation here is that the early $\left(u=t-r^{*}<0\right)$ behavior of the waveform depends only on the initial data of the particle; while the late $(u>0)$ behavior only depends on the black hole characteristics (quasinormal ringing). Here is the challenge for 
Figure 2

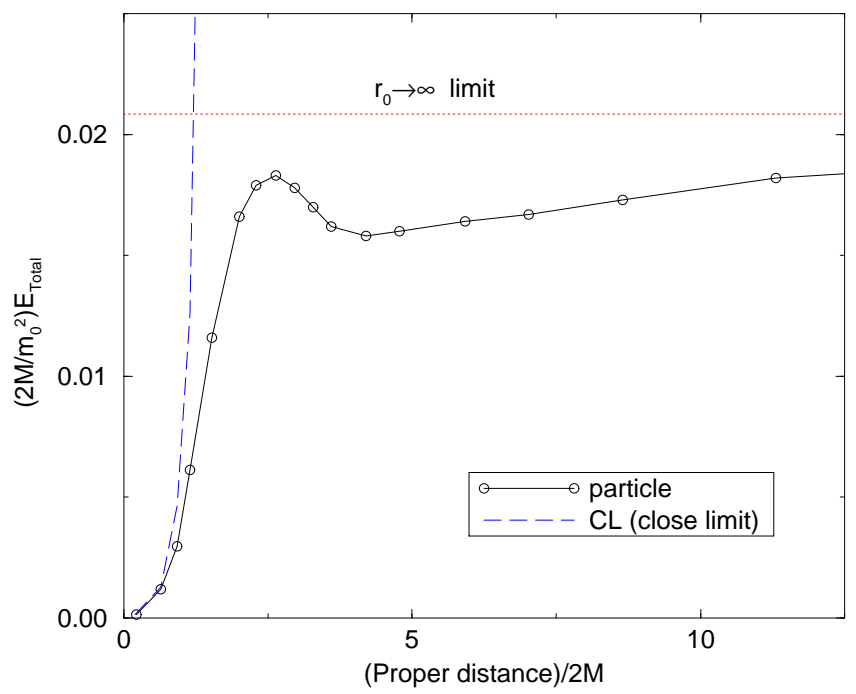

gravitational wave detectors: To have enough sensitivity to resolve this two parts of the waveform and then learn on both, the initial state of motion of the particle and the central black hole.

\section{Observational estimates}

The confirmation of the presence of a black hole with $M=2.4 \times 10^{6} M_{\odot}$ in the center of our galaxy provides an astrophysical scenario for testing our computations. Let us first consider the amplitude of the metric perturbations as the gravitational radiation reaches the earth. From the waveforms given in Fig. 3 and Eq. (2), we find that $\Delta K \sim h \sim 10^{-17}\left(m_{0} / M_{\odot}\right)(8 k p c / r)$, independent of $M$, the mass of the black hole $\mathrm{A}$. The duration of the final burst of radiation can be estimated as $\Delta t \approx 3 \times 10^{-4}\left(M / M_{\odot}\right) s e c \approx 12 \mathrm{~min}$. The next important issue is the frequency of such events. As an order of magnitude estimate we can study the quantity $f=\rho \sigma \nu$, where $\rho \approx 6.5 \times 10^{9} M_{\odot} / p c^{3}$, is the density of stars near the hole, $\sigma_{\text {cap }}=16 \pi M / v^{2}$ is the capture cross section, and $\nu \approx 400 \mathrm{~km} / \mathrm{sec} \approx v$ is the mean velocity of the stars relative to the hole. All

${ }^{a}$ Rotation of the hole and angular momentum of the infalling particle may increase this value in two orders of magnitude 
Figure 3

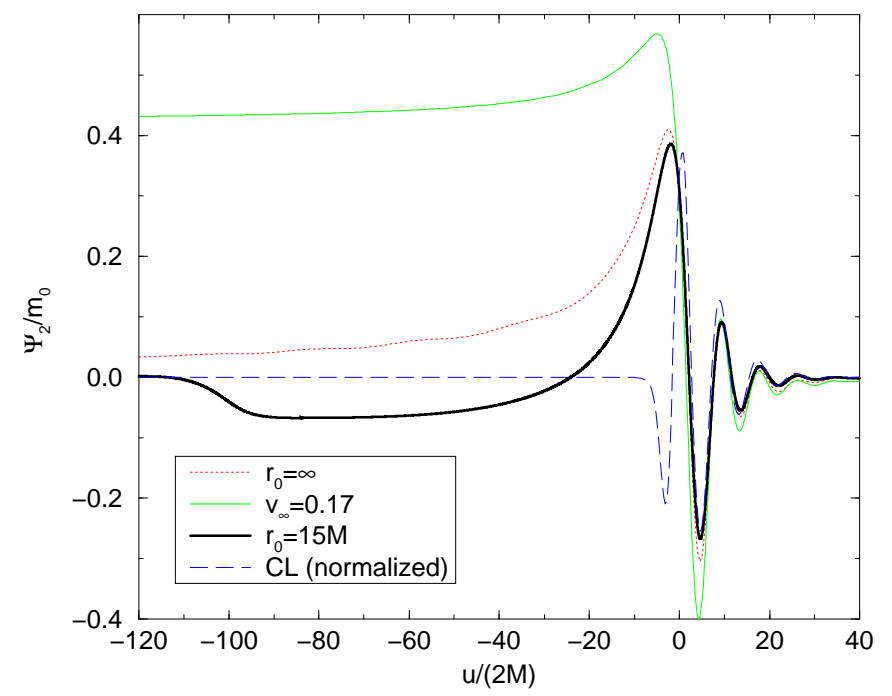

this together gives $f=7.5 \times 10^{-24}\left(M_{\odot} / m_{0}\right)\left(M / M_{\odot}\right)^{2}(c / v) \sec ^{-1} \approx 1$ event/yr. Finally, from the spectrum of gravitational radiation we see that its maximum takes place at a frequency $\omega_{\max } \approx 7 \times 10^{4}\left(M_{\odot} / M\right) \mathrm{Hz} \approx 0.03 \mathrm{~Hz}$. With all this numbers ${ }^{\text {D }}$ in hand we conclude that the galactic center may well be among the first positively detected sources of gravitational radiation (by LISA).

\section{Acknowledgments}

I would like to thank A. Giazotto, J. Horvath, and R. Price for discussions on this problem, and NSF Grant No. PHY0507719 for financial support.

\section{References}

1. C.O. Lousto and R.H. Price, Phys. Rev. D55, 2124 (1997).

2. A. Eckart and R. Genzel, Nature 383, 415 (1996).

${ }^{b}$ The tidal forces of the black hole will disrupt the upper atmosphere of the infalling star (generating a burst of electromagnetic radiation accompanying the gravitational one), but leave its core (where most of the star's mass resides) practically untouched. This justifies our particle approximation. 